\title{
A cultura do sorgo sacarino: revisão
}

\author{
The culture of sweet sorghum: review \\ El cultivo del sorgo sacarino: revisión
}

Recebido: 08/02/2021 | Revisado: 09/02/2021 | Aceito: 09/02/2021 | Publicado: 11/02/2021

Taniele Carvalho de Oliveira

https://orcid.org/ 0000-0002-6900-6449 Universidade do Estado de Mato Grosso, Brasil E-mail: tani.ele@hotmail.com

\begin{abstract}
Resumo
O sorgo sacarino é uma gramínea de grande importância agrícola, apresenta ciclo anual, colmos eretos e suculentos com teores de açúcares elevados, podendo ser cultivada em regiões tropicais e subtropicais do todo o mundo. A cultura se apresenta como uma das fontes renováveis capaz de contribuir para o aumento na produção de etanol, podendo ser usada como cultura complementar à cana-de-açúcar. Nesse contexto, objetivou-se revisar a literatura disponível sobre as potencialidades de cultivo da cultura do sorgo sacarino. O estudo apresentado é de natureza qualitativa e trata-se de uma revisão de literatura e com objetivos de reunir informações sobre o cultivo e utilização de sorgo sacarino e sintetizá-los em um documento, principalmente para uso didático. Diante das observações e pesquisas realizadas foi possível constatar que apesar do sorgo sacarino apresentar potencial para a produção de etanol, a cultura ainda pouco utilizada no país. Essas pesquisas mostraram que a produção de biocombustíveis está em constante evolução, com isso o cultivo de sorgo sacarino auxiliará no desenvolvimento desse cenário. A avaliação de novas possibilidades com o uso do sorgo sacarino na produção de etanol é estrategicamente importante para o país, pois promove o avanço na geração de conhecimentos, melhoria da qualidade dos produtos e incorporação de processos produtivos de menor impacto ambiental, permitindo o fortalecimento do sistema de produção de etanol, bem como a sua diversificação.
\end{abstract}

Palavras-chave: Biocombustível; Etanol; Produtividade; Sorghum bicolor (L.) Moench.

\begin{abstract}
The sweet sorghum is a grass of great agricultural importance, presents an annual cycle, erect and succulent stems with high sugar contents, and can be cultivated in tropical and subtropical regions around the world. The crop presents itself as one of the renewable sources capable of contributing to the increase in ethanol production, and can be used as a complementary crop to sugar cane. In this context, the objective was to review the available literature on the potentialities of the cultivation of sweet sorghum. The study presented is of a qualitative nature and is a literature review with the objective of gathering information on the cultivation and use of sweet sorghum and synthesizing them in a document, mainly for didactic use. Based on the observations and research carried out, it was possible to verify that, although sweet sorghum has potential for ethanol production, the crop is still little used in the country. These researchers showed that the production of biofuels is in constant evolution, so the cultivation of sweet sorghum will assist in the development of this scenario. The evaluation of new possibilities with the use of sweet sorghum in ethanol production is strategically important for the country, because it promotes the advance in the generation of knowledge, improvement of product quality and incorporation of production processes with less environmental impact, allowing the strengthening of the ethanol production system, as well as its diversification.
\end{abstract}

Keywords: Biofuel; Ethanol; Productivity; Sorghum bicolor (L.) Moench.

\section{Resumen}

El sorgo sacarino es una gramínea de gran importancia agrícola, presenta un ciclo anual, tallos erectos y suculentos con alto índice de azúcar, y se puede cultivar en regiones tropicales y subtropicales de todo el mundo. Este cultivo es una de las fuentes renovables capaces de contribuir al aumento de la producción de etanol, y puede utilizarse como cultivo complementario a la caña de azúcar. En este contexto, el objetivo de este estudio es revisar la literatura disponible sobre las potencialidades de cultivo del sorgo sacarino. El estudio que se presenta es de carácter cualitativo y se trata de una revisión bibliográfica con el objetivo de recopilar información sobre el cultivo y uso del sorgo de sacarino y sintetizarla en un documento, principalmente de uso didáctico. A partir de las observaciones e investigaciones realizadas, se pudo comprobar que a pesar de que el sorgo sacarino tiene potencial para la producción de etanol, el cultivo todavía es poco utilizado en el país. Estos estudios han demostrado que la producción de biocombustibles está en constante evolución, por lo que el cultivo de sorgo ayudará al desarrollo de esta escenario. La evaluación de nuevas posibilidades con el uso del sorgo sacarino en la producción de etanol es estratégicamente importante para el país, ya que promueve el avance en la generación de conocimiento, la mejora de la calidad del 
producto y la incorporación de procesos de producción con menor impacto ambiental, permitiendo el fortalecimiento del sistema de producción de etanol, así como su diversificación.

Palabras clave: Biocombustible; Etanol; Productividad; Sorghum bicolor (L.) Moench.

\section{Introdução}

O sorgo sacarino [Sorghum bicolor (L.) Moench] pertence à família das gramíneas, é originária do continente africano, apresenta ciclo anual, colmos eretos e suculentos com teores de açúcar elevados, altura média de 2,5 metros, se adapta a diversos ambientes de cultivo, principalmente em regiões com alta temperatura e deficiência hídrica, que são condições desfavoráveis à maioria dos grãos produzidos (Borém, 2005; Santos et al., 2005).

Diversas pesquisas que indicam a viabilidade de uso do sorgo sacarino na entressafra canavieira no Brasil, proporcionando as usinas a antecipação e ampliação no período de moagem (Teixeira et al., 1997). A cultura se apresenta como uma das fontes renováveis capaz de contribuir para o aumento na produção de etanol, podendo ser usada como cultura complementar à cana-de-açúcar em áreas de reforma, áreas consideradas marginais para a cana ou áreas que não tenham sido contempladas no zoneamento de riscos climáticos da cultura (Emygdio et al., 2011).

O cultivo do sorgo sacarino é uma alternativa economicamente viável para o fornecimento de matéria-prima para destilarias, pois apresenta grande quantidade de açúcares, o que faz dele uma fonte de açúcar e álcool, cerca de $8 \%$ inferior à da cana-de-açúcar, com o ${ }^{\circ}$ brix variando de 16 até 23\% e biomassa entre 40-70 t ha-1 (Olivetti \& Camargo, 1997; Almodares \& Hadi, 2009). Neste contexto, observa-se resultados de 50 a 77 litros de etanol por tonelada de massa verde variando de 80 a $127 \mathrm{~kg}$ de açúcar extraídos por tonelada de massa verde. Sendo possível utilizar a mesma estrutura para colheita e processamento utilizada para cana-de-açúcar (Durães, 2011).

Em avaliações do processamento industrial de sorgo sacarino a cultura obteve um rendimento de até 60 litros de etanol por tonelada de colmos processados. Para a cana-de-açúcar são produzidos, em média, 85 litros de etanol por tonelada (Embrapa, 2013). Semeado na primeira safra, o sorgo sacarino pode fornecer matéria-prima para abastecimento das usinas entre os meses de janeiro a abril, época em que a indústria fica ociosa e a oferta de etanol diminui consideravelmente no país (Fietz et al., 2014).

Nesse contexto, o cultivo de sorgo sacarino apresenta grande potencial de utilização pela indústria de produção de álcool devido as vantagens que viabilizam seu cultivo. Com isso, objetivou-se com o presente trabalho revisar a literatura disponível sobre as potencialidades de cultivo da cultura do sorgo sacarino.

\section{Metodologia}

O estudo apresentado é de natureza qualitativa e trata-se de uma revisão de literatura e com objetivos de reunir informações sobre o cultivo e utilização de sorgo sacarino e sintetizá-los em um documento, principalmente para uso didático. Para isso foi feita uma pesquisa sistemática e ordenada em bases em dados eletrônicos (periódicos científicos, documentos técnicos e livros especializados no assunto), conforme Pereira et al. (2018).

\section{Origem e Domesticação}

A cultura do sorgo originou-se na África, onde atualmente se encontra a maior variabilidade em espécies silvestres e cultivadas (Figura 1). Relatos que sua domesticação ocorreu provavelmente na Etiópia, através da seleção de espécies silvestres (Sorghum arundinaceum ou Sorghum vercilliflorum) aproximadamente há 7000 anos (Santos et al., 2005). 
Figura 1. Origem e domesticação de algumas espécies cultivadas.

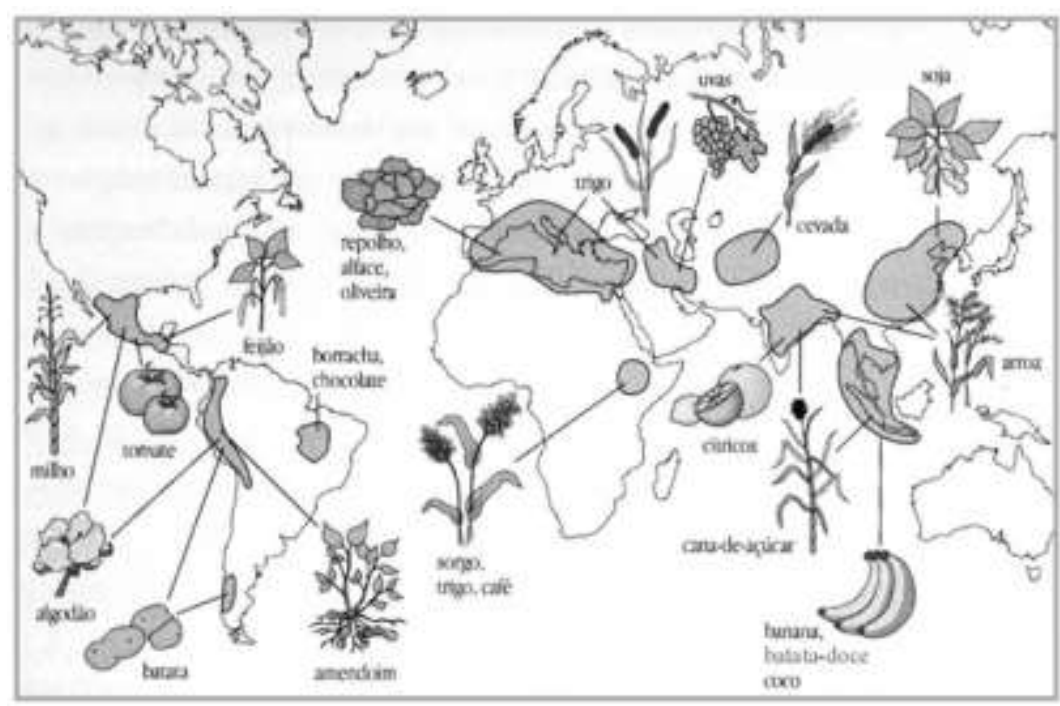

Fonte: Primack \& Rodrigues (2001).

O sentido da seleção foi de tipos silvestres de sementes pequenas e quebradiças para sementes maiores e robustas. Essa seleção resultou em plantas com características muito diferentes em altura, tipo de inflorescência, e utilização final (alimentação, forragem, grãos, etc.) (Olembo et al., 2010).

O sorgo foi distribuído do nordeste da África para o continente através do transporte e do comércio pelas rotas do Oriente Médio para a Índia. Da Índia, acredita-se ter sido levado para a China ao longo da rota da seda e através de navegação de cabotagem para o Sudeste Asiático. Da África Ocidental o sorgo foi levado para as Américas através do tráfico de escravos e introduzido nos Estados Unidos para o cultivo comercial no final do século XIX. Posteriormente, foi introduzido na América do Sul e Austrália. Sendo amplamente cultivada em áreas mais secas da África, Ásia, Américas, Europa e Austrália entre as latitudes de até $50^{\circ} \mathrm{N}$ na América do Norte e da Rússia e $40^{\circ} \mathrm{S}$, na Argentina (Balole \& Legwaila, 2006).

No Brasil, a sua introdução se atribui aos escravos, onde a cultura ficou conhecida como milho d'Angola (Lira, 1981), e é considerada uma cultura bastante recente, a partir da década de 60 que começou a ser conhecido (Olivetti \& Camargo, 1997). Somente no final do século XIX que apresentou importância dentre os cereais, passando a ser o quinto do mundo em área cultivada, após o trigo, milho, arroz e cevada (Kill \& Menezes, 2005).

\section{Morfologia e Aspectos Gerais}

O sorgo pertence à Família Poaceae (Gramíneas); Gênero Sorghum; Espécie: Sorghum bicolor. A espécie Sorghum bicolor (L.) Moench é uma espécie diplóide com $2 \mathrm{n}=20$ cromossomos (Santos et al., 2005). O sistema radicular é fibroso que se ramifica profusamente. As raízes possuem sílica na endoderme, grande quantidade de pelos absorventes e altos índices de lignificação de periciclo (Durães, 2011) e sob condições favoráveis, os nós acima do solo podem produzir raízes adventícias fortes, que ajudam a ancorar a planta (Bassam, 2010).

O caule é cilíndrico e ereto, dividido em nós e entrenós, cada entrenó tem a capacidade de desenvolver um sistema radicular independente, ainda que permaneça ligado à da haste principal (Figura 2). A altura da planta varia entre os $0,4 \mathrm{e} 5 \mathrm{~m}$, e a largura, na base, entre 1,5 a 5 cm de diâmetro (Magalhães et al., 2008; Bassam, 2010; May et al., 2013). Algumas espécies podem acumular níveis elevados de açúcar no parênquima do colmo (Quintero et al., 2012) e o caldo extraído contém altos níveis de sacarose e açúcar invertido que são facilmente fermentados para produção de etanol (Prasad et al., 2007). 
Figura 2. Espécie Sorghum bicolor (L.) Moench.

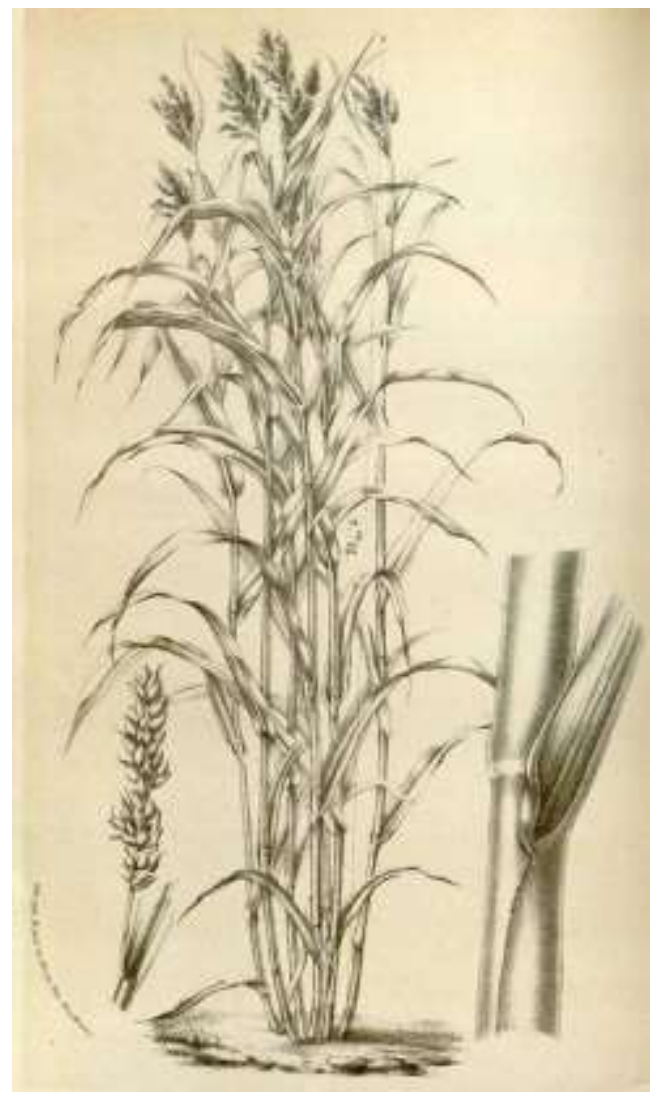

Fonte: Van Houtte (1854)

As folhas são alternadas e as bainhas são compridas sobrepondo-se umas às outras, apresentam folhas com diferentes comprimentos, sendo que durante o período da seca as folhas enrolam-se contribuindo para reduzir a transpiração (Campos et al., 1973).

É uma planta autógama, com baixa taxa de fecundação cruzada que varia conforme o ambiente e o genótipo, de 5 a 20\%. Possuem flores completas e em geral não apresenta autoesterilidade (Rooney, 2004; Borém, 2005). A inflorescência é composta por uma panícula com cachos em ráquis terciárias, cada uma com uma ou várias espiguetas. Algumas espiguetas são sésseis e outras são pediceladas, exceto as espiguetas sésseis terminal, o qual é acompanhado por duas espiguetas pediceladas. As espiguetas sésseis têm ambos os sexos, masculino (androceu) e feminino (gineceu), e as pediceladas são geralmente do sexo masculino ou do sexo feminino (Reddy et al., 2008). O fruto é uma cariopse ou grão seco, podendo apresentar coloração preta, marrom, marrom clara, vermelha, bronze, amarela, creme, cinza e branca (Mapa, 1997).

A planta apresenta metabolismo $\mathrm{C} 4$, resposta fotoperiódica típica de dias curtos e altas taxas fotossintéticas (Magalhães, 2000), embora a cultura seja nativa dos trópicos, possui boa adaptação em regiões temperadas e tropicais. No Brasil, o sorgo tem despontado como alternativa para diversas regiões, pois se adapta a diversos ambientes, principalmente onde ocorre deficiência hídrica, temperaturas altas, podendo também ser cultivada em solos ácidos e alcalinos (Santos et al., 2005).

Agronomicamente o sorgo é classificado em 5 grupos distintos: Sorgo granífero que inclui tipos de sorgo de porte baixo, altura de planta até $170 \mathrm{~cm}$, que produz na extremidade superior, uma panícula (cacho), onde se localizam os grãos (principal produto deste tipo de sorgo); Sorgo forrageiro que compreende um tipo de sorgo de porte alto, com altura de planta superior a dois metros, muitas folhas, panículas abertas, com poucas sementes, elevada produção de forragem (variedades de Sorghum bicolor e capim sudão ou híbridos inter específicos destes, ou seja, Sorghum bicolor x Sorghum sudanense); Sorgo 
sacarino possui porte elevado sua altura é superior a dois metros, caracterizado principalmente por apresentar colmo doce e suculento apropriados para produção de açúcar e álcool e/ou confecção de silagem; Sorgo vassoura inclui tipos de cujas panículas são confeccionadas vassouras (Ribas, 2000); e o Sorgo biomassa são plantas de porte alto, podendo atingir mais de 5 m de altura, sua principal finalidade é produção de biomassa utilizada como fonte renovável de energia (May et al., 2013).

\section{Potencialidades de Cultivo}

O sorgo sacarino apresenta colmos com caldo semelhante ao da cana-de-açúcar, rico em açúcares fermentescíveis, e pode ser utilizado para a produção de etanol utilizando a mesma instalação das usinas da cana-de-açúcar (Figura 3) (Durães, 2011). Seu cultivo permite a otimização da produção de biocombustíveis pela utilização complementar da área cultivada com cana-de-açúcar, devido á complementariedade temporal destas culturas ocasionada pela expansão do setor produtivo (Cunha \& Severo Filho, 2010).

Figura 3. Comparação das características de produção da cana-de-açúcar e sorgo sacarino.

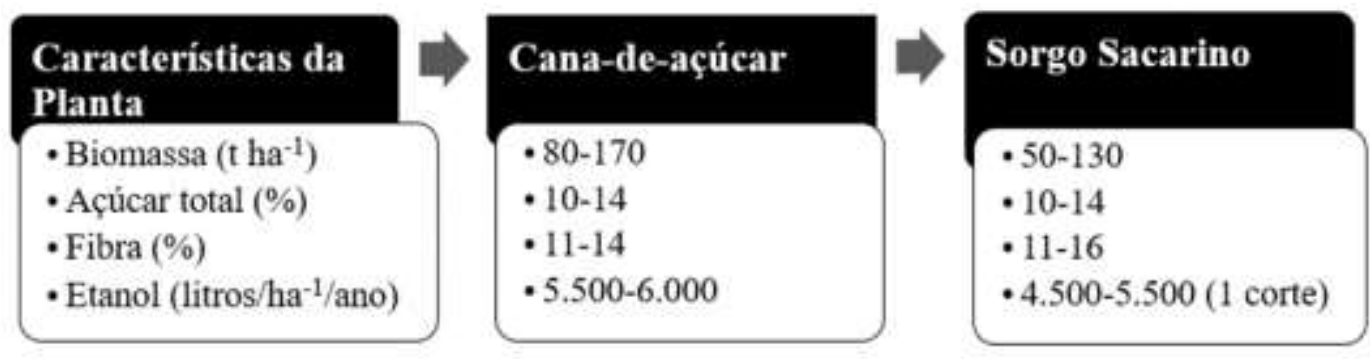

Fonte: Canavialis (2010)

A expansão da cultura de sorgo sacarino no Brasil tem amplas perspectivas e admite modelos diferenciados. O modelo de expansão preferencial da cultura de sorgo sacarino é na entressafra da cana-de-açúcar ou renovação de canaviais, objetivando complementar a produção de etanol, antecipando a oferta de matéria-prima de qualidade, visando competitividade com sustentabilidade, aumentos de produtividade e redução de custos de produção e maior eficiência do uso de recursos e insumos (Durães et al., 2012).

As usinas têm a possibilidade de iniciar suas atividades industriais com o sorgo sacarino a partir da segunda quinzena de março, visto que a janela ideal de plantio de sorgo é de 15 de novembro a 15 de janeiro, período em que essa cultura encontra as melhores condições de desenvolvimento, proporcionando, assim, um aumento na amplitude da janela de colheita. O potencial mínimo de geração de etanol de sorgo sacarino está estimado em $3.223 \mathrm{~L} \mathrm{ha}^{-1}$, com uma produtividade que gira em torno de $55 \mathrm{t} \mathrm{ha}^{-1}$, o que representa cerca de $58,6 \mathrm{~L} \mathrm{t}$ (Assis \& Moraes, 2014).

Para que a cultura expresse todo seu potencial no campo e consequentemente alta produtividade de biomassa e elevada produção de caldo por hectare cultivado, a lavoura de sorgo sacarino demanda os mesmos preceitos recomendados para qualquer cultura, como adequado preparo de solo, boa fertilização de base e cobertura, controle de plantas daninhas e pragas. A recomendação de semeio é mesma para a maioria das áreas produtoras de cana (regiões Centro-Oeste e Sudeste), e a colheita é programada para março e abril, justamente quando a cana ainda não apresenta elevados valores de ${ }^{\circ}$ Brix, inviabilizando seu corte (May, 2011).

A utilização do sorgo sacarino apresenta algumas vantagens para a indústria, como matéria prima de qualidade durante todo o ano, aumentando o período de processamento e produção de etanol/açúcar, e otimizando o uso da estrutura e consequentemente o capital investido (Figura 4) (CanaVialis, 2010; Embrapa, 2013). 
Figura 4. Comparativo entre as características cana-de-açúcar e sorgo sacarino.

\begin{tabular}{|c|c|c|}
\hline $\begin{array}{l}\text { Características da } \\
\text { Cultura }\end{array}$ & Cana-de-açúcar & Sorgo Sacarino \\
\hline $\begin{array}{l}\text { - Plantio } \\
\text { - Necessidade } \\
\text { - Ciclo } \\
\text { - Cortes/ano } \\
\text { - Custo/ha (RS) }\end{array}$ & $\begin{array}{l}\cdot \text { Muda } \\
\cdot 7-10 \text { ton talo } \text { ha }^{-1} \\
\cdot 11-12 \text { meses } \\
\cdot 1 \\
\cdot 5.500\end{array}$ & $\begin{array}{l}\cdot \text { Semente } \\
\cdot 5-10 \mathrm{~kg} \text { sementes ha- } \\
\cdot 115-130 \text { dias } \\
\cdot 1-2 \\
\cdot 1.100\end{array}$ \\
\hline
\end{tabular}

Fonte: CanaVialis (2010)

Já para o campo é uma excelente alternativa na área de renovação de cultivo e principalmente mantendo o foco na produção de etanol, uma cultura "start" para novas usinas na área de expansão onde está ocorrendo o processo de plantio e multiplicação dos canaviais até que a totalidade da área seja preenchida com cana, e substituição da cana em área proibitivas, locais com topografia, clima e solos onde a cana não se desenvolve (CanaVialis, 2010; Embrapa, 2013).

Neste contexto, o cultivo de sorgo sacarino demonstra alto potencial para fins de produção de etanol, porém ressaltase a importância do desenvolvimento de mais pesquisas para conferir uma melhor adaptabilidade ao mesmo com ganho na produção, e também necessidade de fomento para inserção do sorgo sacarino nos diferentes sistemas de produção agrícola ou ainda em áreas de pastagens degradas.

\section{Considerações Finais}

Diante das observações e pesquisas realizadas foi possível constatar que apesar do sorgo sacarino apresentar potencial para a produção de etanol, a cultura ainda pouco utilizada no país. Essas pesquisas mostraram que a produção de biocombustíveis está em constante evolução, com isso o cultivo de sorgo sacarino auxiliará no desenvolvimento desse cenário.

Dentre as estratégias para maximizar ou ampliar a capacidade produtiva, a avaliação de novas possibilidades como o uso do sorgo sacarino na produção de etanol é estrategicamente importante para o país, pois promove o avanço na geração de conhecimentos, melhoria da qualidade dos produtos e incorporação de processos produtivos de menor impacto ambiental, permitindo o fortalecimento do sistema de produção de etanol, bem como a sua diversificação.

\section{Referências}

Almodares, A., \& Hadi, M. R. (2009). Production of bioethanol from sweet sorghum: A review. African Journal of Agricultural Research, 4(9), 772-780.

Assis, R. T., \& Morais, C. G. (2014). Sorgo sacarino, a segunda safra do etanol no Brasil. Instituto de Ciências da Saúde, Agrárias e Humanas (ISAH). Recuperado de http://site.uniaraxa.edu.br/wp-content/uploads/2014/09/sorgo-sacarino-a-segunda-safra-do-etanol-no-brasil.pdf.

Balole, T. V., \& Legwaila, G. M. (2006). Sorghum bicolor (L.) Moench. Recuperado de http://www.prota4u.org/protav8.asp?h=M11,M26,M6\&t=origin,sorghum\&p=Sorghum+bicolor\#Description.

Bassam, N. (2010). Handbook of bioenergy crops - A complete reference to species, development and applications. London: Earthscan.

Borém, A. (2005). Melhoramento de espécies cultivadas. Viçosa: UFV.

Campos, T., \& Canéchio Filho, V. (1973). Principais culturas II. Campinas: IAC.

CanaVialis - Monsanto Company. (2010). Sorgo Sacarino. Recuperado de http://www.canavialis.com.br/produtos/sorgo-sacarino/index.aspx

Cunha, S. P., \& Severo Filho, W. A. (2010). Avanços tecnológicos na obtenção de etanol a partir de sorgo sacarino (Sorghum bicolor (L.) Moench). TecnoLógica, 14(2), 69-75.

Durães, F. O. (2011). Sorgo sacarino: desenvolvimento de tecnologia agronômica. Revista Agroenergia, 2 (3), 7.

Durães, F. O. M., May, A., \& Parrella, R. D. C. (2012). Sistema agroindustrial do sorgo sacarino no Brasil e a participação público-privada: oportunidades, perspectivas e desafios. Sete Lagoas: Embrapa Milho e Sorgo. 
Embrapa Milho e Sorgo. (2013). Sistemas de Produção, 2 Versão Eletrônica - $3^{a}$ edição. Recuperado de http://www.cnpms.embrapa.br/publicacoes/sorgo/index.htm.

Emygdio, B. M., Rosa, A. P. S. A., Oliveira, A. C. B., Parrella, R. D. C., Schaffert, R. E., \& May, A. (2011). Desempenho de cultivares de sorgo sacarino para a produção de etanol sob diferentes densidades de plantas. Embrapa Clima Temperado-Boletim de Pesquisa e Desenvolvimento (INFOTECA-E).

Fietz, C. R., Lima Filho, O. F., Silva, C. J., Comunello, E., \& Flumignan, D. (2014). Sorgo sacarino: época de semeadura, com base no risco climático, na Região Sul de Mato Grosso do Sul. Embrapa Agropecuária Oeste-Circular Técnica (INFOTECA-E).

Kiill, L. H. P., \& Menezes, E. A. (2005). Espécies vegetais exóticas com potencialidades para o semi-árido brasileiro. Embrapa informação tecnológica.

Lira, M. D. A. (1981). Considerações sobre o potencial do sorgo em Pernambuco. Curso de extensão sobre a cultura do sorgo, 87-88.

Magalhães, P. C., Durães, F. O. M., \& Rodrigues, J. A. S. (2008). Cultivo de sorgo: ecofisiologia. Recuperado de http://www.cnpms.embrapa.br/publicacoes/sorgo_4_ed/ecofisiologia.htm.

Magalhães, P. C., Durães, F. O. M., \& Schaffert, R. E. Fisiologia da planta do sorgo. Sete Lagoas: Embrapa Milho e Sorgo.

Mapa - Ministério da Agricultura, Pecuária e Abastecimento. (1997). Descritores mínimos de sorgo (Sorghum spp.).

May, A., Silva, D. D., \& Santos, F. C. (2013). Cultivo do sorgo biomassa para a cogeração de energia elétrica. Embrapa Milho e Sorgo-Documentos (INFOTECA-E).

Olembo, K. N., M'mboyi, F., Kiplagat, S., Sitieney, J. K., \& Oyugi, F. K. (2010). Sorghum breeding in sub-Saharan Africa: the success stories. African Biotechnology Stakeholders Forum (ABSF).

Olivetti, M. P. A., \& Camargo, A. M. M. P. (1997). Aspectos econômicos e desenvolvimento da cultura do sorgo. Informações Econômicas-Governo do Estado de São Paulo Instituto de Economia Agrícola, 27, 248-251.

Pereira, A. S., Shitsuka, D. M., Parreira, F. J. \& Shitsuka, R. (2018). Metodologia da Pesquisa Científica. UFSM NTE.

Prasad, S., Singh, A., Jain, N., \& Joshi, H. C. (2007). Ethanol production from sweet sorghum syrup for utilization as automotive fuel in India. Energy \& Fuels, 21(4), 2415-2420.

Primack, R. B., \& Rodrigues, E. (2001). Biologia da Conservação. Londrina: E. Rodrigues.

Quintero, V. P., López, J. L. A., Colmenero, A. Z., García, N. M., Colín, C. N., Bonilla, J. L. S., Rangel, M. R. A., \& Prom, L. (2012). Genetic diversity of sweet sorghum germplasm in Mexico using AFLP and SSR markers. Pesquisa Agropecuária Brasileira, 47(8), 1095-1102.

Reddy, B. V. S., Ramesh, S., Kumar, A. A., \& Gowda, C. L. L. (2008). Sorghum Improvement in the New Millennium. India: ICRISAT.

Rooney, W. L. (2004). Sorghum Improvement - Integrating Traditional and New Technology to Produce Improved Genotypes. Advances in Agronomy, 83, 37 109.

Santos, F. G., Casela, C. R., \& Waquil, J. M. (2005). Melhoramento de Sorgo. In: Borém, A. (org). Melhoramento de Espécies Cultivadas. Viçosa: UFV.

Teixeira, C. G., Jardine, J. G., \& Beisman, D. A. (1997). Utilização do sorgo sacarino como matéria-prima complementar à cana-de-açúcar para obtenção de etanol em microdestilaria. Food Science and Technology, 17(3), 248-251.

Van Houtte, L.B. (1854). Flore des serres et des jardin de l'Europe (1845-1880). Serres. http://www.plantillustrations.org/illustration.php?id_illustration=52134\&id_taxon=10149\&mobile=0\&SID=3h52sbkjurpv381sgssrnnnk9a\&language=English $\&$ thumbnails_selectable $=0 \&$ selected_thumbnail $=0 \&$ query_type $=$ species\&query_broad_or_restricted=broad $\&$ group $=0 \&$ lay_out $=0 \& u h d=0$ 